\title{
THE PLANE PROBLEM OF COLLINEAR RIGID LINES UNDER ARBITRARY LOADS
}

\author{
JIANG CHI-PING \\ Institute of Mechanics, Chinese Academy of Sciences, Beijing 100080, China
}

\begin{abstract}
The elastic plane problem of collinear rigid lines under arbitrary loads is dealt with. Applying the Riemann-Schwarz symmetry principle integrated with the analysis of the singularity of complex stress functions, the general formulation is presented, and the closed-form solutions to several problems of practical importance are given, which include some published results as the special cases. Lastly the stress distribution in the immediate vicinity of the rigid line end is cxamined.
\end{abstract}

\section{INTRODUCTION}

THE PRESENCE of inclusions plays an important role in the fracture behavior of engineering materials, cspccially compositcs. In analysing such problems, certain idealizations concerning the geometry and the mechanical properties of the constituent materials are usually made in order to be tractable mathematically. For an elastic plane problem, if the value of the elastic modulus of a flat inclusion is much greater than that of the matrix is, it appears reasonable to consider it as a rigid line. Furthermore, from the viewpoint of inhomogeneities in solids, a rigid line and a slit crack are the two extreme cases of a flat inhomogeneity, namely, for a rigid line $E \rightarrow \infty$, and for a crack $E \rightarrow 0$, where $E$ is the Young's modulus. Since Eshelby's paper[1], some investigations on the rigid line inhomogeneity problem have been made, however, because of the analytic difficulties involved, the number of closed solutions for particular geometries and loading conditions is very limited. In ref. [2], the solution for an elastic plane containing a rigid line under an arbitrary uniform stress state at infinity can be found. Recently, the elastic plane problem of collinear periodical rigid lines was dealt with by Hao and $\mathfrak{W} u[3]$, who considered only a special loading case in which the tension stress at infinity is parallel to the rigid lines.

In the following an attempt is made to find the solution for an elastic plane with collinear rigid lines under most general loading conditions which include both an arbitrary uniform stress state at infinity and a concentrated load at an arbitrary point. Applying the Riemann-Schwarz symmetry principle integrated with the singularity of complex stress functions[4], the general formulation is presented, and the closed solutions to several problems of practical importance are given. Especially, the closed solution for a concentrated load at an arbitrary point is obtained. In addition to providing solutions to such practical problems as wedge loading or rivet loading at an arbitrary angle, this solution can be used as Green's function to obtain the stresses in the elastic plane with any given distribution of loads.

\section{GENERAL FORMULATION OF THE PROBLEM}

To formulate the problem we use the complex stress functions $\Phi(z)$ and $\Psi(z)$, in terms of which the stresses $\left(\sigma_{x}, \sigma_{y}\right.$ and $\left.\tau_{x y}\right)$ and displacements $(u, v)$ are given as[2]:

$$
\begin{gathered}
\sigma_{x}+\sigma_{y}=2[\Phi(z)+\overline{\Phi(z)}] \\
\sigma_{y}-i \tau_{x y}=\Phi(z)+\overline{\Phi(z)}+z \overline{\Phi^{\prime}(z)}+\overline{\Psi(z)} \\
2 \mu\left(u^{\prime}+i v^{\prime}\right)=\kappa \Phi(z)-\overline{\Phi(z)}-\overline{\Phi^{\prime}(z)}-\overline{\Psi(z)}
\end{gathered}
$$

where

$$
u^{\prime}=\frac{\partial u}{\partial x} \quad v^{\prime}=\frac{\partial v}{\partial x}
$$


$\mu$ is the shear modulus and $\kappa=3-4 v$ for plane strain, $\kappa=(3-v) /(1+v)$ for generalized plane stress, $v$ being Poisson's ratio. $\Phi(z)$ and $\Psi(z)$ are holomorphic in the whole region occupied by the elastic body but poles at concentrated load points. Assuming that a concentrated force $X+i Y$ and a moment $m$ are applied at an arbitrary point $z_{0}$, we have[2]

$$
\begin{gathered}
\Phi(z)=\frac{M}{z-z_{0}}+\Phi_{0}(z) \\
\Psi(z)=\frac{N}{z-z_{0}}+\frac{\bar{z}_{0} M+M_{0}}{\left(z-z_{0}\right)^{2}}+\Psi_{0}(z)
\end{gathered}
$$

where $\Phi_{0}(z)$ and $\Psi_{0}(z)$ are holomorphic in the whole region which is occupied by the elastic body, and

$$
M=-\frac{X+i Y}{2 \pi(1+\kappa)}, \quad N=\frac{\kappa(X-i Y)}{2 \pi(1+\kappa)}, \quad M_{0}=-\frac{m}{2 \pi}
$$

By applying the Riemann-Schwarz symmetry principle, a new analytic function $\Omega(z)$ can be defined:

$$
\Omega(z)=\bar{\Phi}(z)+z \bar{\Phi}^{\prime}(z)+\bar{\Psi}(z)
$$

where $\bar{\Phi}(z)=\overline{\Phi(\bar{z})}$. It is obvious that $\Omega(z)$ can be expressed as:

$$
\Omega(z)=\frac{\bar{N}}{z-\bar{z}_{0}}+\frac{\left(z_{0}-\bar{z}_{0}\right) \bar{M}+\bar{M}_{0}}{\left(z-\bar{z}_{0}\right)^{2}}+\Omega_{0}(z)
$$

where $\Omega_{0}(z)$ is holomorphic in the region in which $\Omega(z)$ is defined. Thus, (2.2) and (2.3) can be rewritten as

$$
\begin{gathered}
\sigma_{y}-i \tau_{x y}=\Phi(z)+\Omega(\bar{z})+(z-\bar{z}) \overline{\Phi^{\prime}(z)} \\
2 \mu\left(u^{\prime}+i v^{\prime}\right)=\kappa \Phi(z)-\Omega(\bar{z})-(z-\bar{z}) \overline{\Phi^{\prime}(z)} .
\end{gathered}
$$

Now consider our problem.

In an infinite elastic plane, a series of collinear rigid lines are placed along a part, $L$, of the real axis where $L$ is a union of straight-line segments $L_{j}$ with the end points $a_{j}$ and $b_{j}(j=1, \ldots, n)$ (Fig. 1). A concentrated force $X+i Y$ and a moment $m$ are applied at an arbitrary point $z_{0} . \sigma_{1}$ and $\sigma_{2}$ are the principal stresses at infinity and $\alpha$ is the angle between $\sigma_{1}$ and $0 x$ axis. Then the boundary conditions for the problem may be expressed as follows;

$$
(u+i v)^{+}=(u+i v)^{-}=\left\{\begin{array}{lll}
u_{1}+i\left[v_{1}+c_{1}\left(x-x_{1}\right)\right] & \text { on } & L_{1} \\
\cdots \cdots & & \\
u_{n}+i\left[v_{n}+c_{n}\left(x-x_{n}\right)\right] & \text { on } & L_{n}
\end{array}\right.
$$

where $u_{j}$ and $v_{j}$ are displacements of the midpoint $\left(x_{j}, 0\right)$ of the rigid line $L_{j}$ and $\epsilon_{j}$ the counterclockwise rotation of $L_{j}(j=1, \ldots, n)$, superscripts + and - refer to the value of the

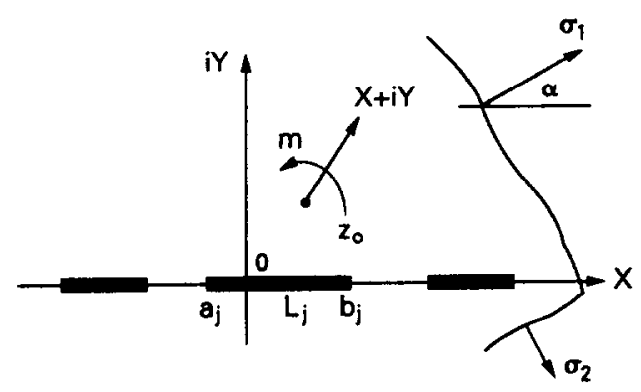

Fig. 1. Infinite plane with collinear rigid lines under a concentrated load at an arbitrary point and a uniform stress state at infinity. 
functions on the real axis as approached from the upper half-plane and the lower half-plane, respectively. Taking the derivative of $(2.12)$ with respect $x$, we obtain

$$
\begin{gathered}
\left(u^{\prime}+i v^{\prime}\right)^{+}=\left(u^{\prime}+i v^{\prime}\right)^{-}=H\left(\epsilon_{1}, \ldots, \epsilon_{n}\right) \text { on } L \\
H\left(\epsilon_{1}, \ldots, \epsilon_{n}\right)=\left\{\begin{array}{lll}
\epsilon_{1} i & \text { on } L_{1} \\
\ldots & \ldots & \\
\epsilon_{n} i & \text { on } & L_{n}
\end{array}\right.
\end{gathered}
$$

In addition, to determine solely the solution of the problem, the equilibrium conditions of rigid lines must be considered. Assuming that rigid lines are traction-free, we have

$$
\begin{array}{ll}
\int_{a_{j}}^{b_{j}}\left(\sigma_{y}-i \tau_{x y}\right)^{+} \mathrm{d} x-\int_{a_{j}}^{b_{j}}\left(\sigma_{y}-i \tau_{x y}\right)^{-} \mathrm{d} x=0 & j=1, \ldots, n \\
\int_{a_{j}}^{b_{j}} x \sigma_{y}^{+} \mathrm{d} x-\int_{a_{j}}^{b_{j}} x \sigma_{y}^{-} \mathrm{d} x=0 & j=1, \ldots, n .
\end{array}
$$

Substituting (2.11) into (2.13), we obtain

$$
\begin{array}{ll}
\kappa \Phi^{+}(t)-\Omega^{-}(t)=2 \mu H & \text { on } L \\
\kappa \Phi^{-}(t)-\Omega^{+}(t)=2 \mu H & \text { on } L
\end{array}
$$

where $t$ denotes the point on the real axis.

The addition and subtraction of (2.17) and (2.18) yield

$$
\begin{aligned}
& {[\kappa \Phi(t)-\Omega(t)]^{+}+[\kappa \Phi(t)-\Omega(t)]^{-}=4 \mu H \text { on } L} \\
& {[\kappa \Phi(t)+\Omega(t)]^{+}-[\kappa \Phi(t)+\Omega(t)]=0 \text { on } L .}
\end{aligned}
$$

Referring to (2.5) and (2.9), $\Phi(z)$ and $\Omega(z)$ in the present problem can be written as

$$
\begin{gathered}
\Phi(z)=\frac{M}{z-z_{0}}+\Gamma+\Phi_{0}(z) \\
\Omega(z)=\frac{\bar{N}}{z-\bar{z}_{0}}+\frac{\bar{M}\left(z_{0}-\bar{z}_{0}\right)+\bar{M}_{0}}{\left(z-\bar{z}_{0}\right)^{2}}+\bar{\Gamma}+\bar{\Gamma}^{\prime}+\Omega_{0}(z)
\end{gathered}
$$

where $\Phi_{0}(z)$ and $\Omega_{0}(z)$ are holomorphic functions in the whole plane cut along $L$ and they vanish at infinity, and

$$
\operatorname{Re} \Gamma=\frac{1}{4}\left(\sigma_{1}+\sigma_{2}\right), \quad \Gamma^{\prime}=-\frac{1}{2}\left(\sigma_{1}-\sigma_{2}\right) e^{-2 i \alpha}
$$

Substituting (2.21) and (2.22) into (2.20), we obtain

$$
\left[\kappa \Phi_{0}(t)+\Omega_{0}(t)\right]^{+}-\left[\kappa \Phi_{0}(t)+\Omega_{0}(t)\right]^{-}=0 \text { on } L .
$$

According to Liouville's theorem, we have on the whole plane

$$
\kappa \Phi_{0}(z)+\Omega_{0}(z)=0 .
$$

Substituting (2.21) and (2.22) into (2.19) and using (2.25), we obtain

$$
\Phi_{0}^{+}(t)+\Phi_{0}^{-}(t)=f(t) \text { on } L
$$

where

$$
f(t)=-\frac{M}{t-z_{0}}+\frac{N}{\kappa\left(t-\bar{z}_{0}\right)}+\frac{M\left(z_{0}-\bar{z}_{0}\right)+\bar{M}_{0}}{\kappa\left(t-\bar{z}_{0}\right)^{2}}-\Gamma+\frac{\Gamma}{\kappa}+\frac{\Gamma^{\prime}}{\kappa}+\frac{2 \mu H}{\kappa}
$$


The general solution of eq. (2.26) can be expressed as[2]

$$
\Phi_{0}(z)=\frac{X_{0}(z)}{2 \pi i} \int_{L} \frac{f(t)}{X_{0}^{+}(t)} \frac{\mathrm{d} t}{t-z}+X_{0}(z) P(z)
$$

where

$$
X_{0}(z)=\prod_{j=1}^{n}\left(z-a_{j}\right)^{-1 / 2}\left(z-b_{j}\right)^{-1 / 2}
$$

which is a single-valued branch in the plane cut along $L$ and for which

$$
\lim _{|z| \rightarrow \infty} z^{n} X_{0}(z)=1
$$

$P(z)$ is an arbitrary polynomial consistent with the behavior of $\Phi_{0}(z)$ at infinity:

$$
P(z)=C_{1} z^{n-1}+C_{2} z^{n-2}+\cdots+C_{n} .
$$

The integration constants $C_{1}, \ldots, C_{n}$ and the rigid line rotations $\epsilon_{1}, \ldots, \epsilon_{n}$ are determined from the equilibrium conditions, (2.15) and (2.16), of rigid lines.

Substituting (2.10) into (2.15), we can obtain

$$
\int_{a_{j}}^{b_{j}}[\Phi(t)-\Omega(t)]^{+} \mathrm{d} t-\int_{a_{j}}^{b_{j}}[\Phi(t)-\Omega(t)]^{-} \mathrm{d} t=0 \quad j=1, \ldots, n
$$

which can be reduced to integrals along closed contours:

$$
\oint_{\Lambda_{j}}[\Phi(z)-\Omega(z)] \mathrm{d} z=0 \quad j=1, \ldots, n
$$

where $\Lambda_{j}$ are clockwise closed contours encircling the rigid lines $L_{j}$ with poles $\left(z_{0}, \bar{z}_{0}\right)$ out.

Similarly, the substitution of (2.10) into (2.16) yields

$$
\operatorname{Re} \oint_{\Lambda_{j}} z[\Phi(z)-\Omega(z)] \mathrm{d} z=0 \quad j=1, \ldots, n .
$$

The set of $2 n$ linear algebraic equations given by eqs (2.33) and (2.34) determine the remaining complex constants $C_{1}, \ldots, C_{n}$ and real constants $\epsilon_{1}, \ldots, \epsilon_{n}$. On the other hand, for large value of $|z|$ we have[2]

$$
\Phi(z)=\Gamma+\frac{M}{z}+0\left(\frac{1}{z^{2}}\right)
$$

From (2.21), (2.28) and (2.35) it is seen that

$$
C_{1}=0 \text {. }
$$

Obviously, (2.36) may be used instead of one of eqs (2.33).

The above method is also efficient to solve the problem of collinear periodical rigid lines if we make use of the following conformal transformation of the $z$-plane onto the $\zeta$-plane:

$$
z=\frac{2 b}{\pi} \operatorname{arctg} \zeta
$$

\section{SEVERAL CLOSED SOLUTIONS}

In the following we consider the application of the solution given by eq. (2.28) to various geometries and loading conditions which may have some practical importance.

\section{Example 1}

Consider a rigid line under a concentrated force $X+i Y$ and a moment $m$ at an arbitrary point $z_{0}$, and an arbitrary uniform stress state at infinity (Fig. 2). 


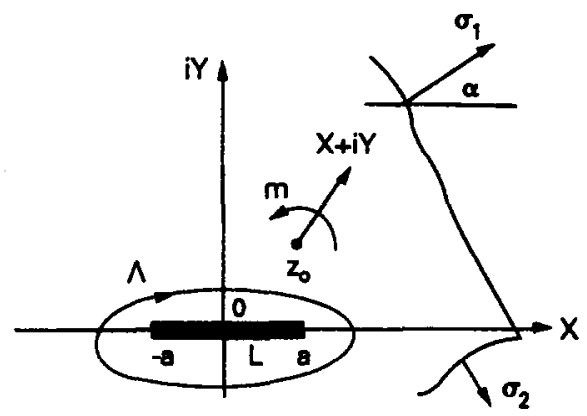

Fig. 2. Infinite plane with a rigid line.

Noting (2.36), in this case, $(2.28)$ becomes

$$
\Phi_{0}(z)=I(z) X_{0}(z)
$$

where

$$
I(z)=\frac{1}{2 \pi i} \int_{-a}^{a} \frac{f(t)}{X_{0}^{+}(t)} \frac{\mathrm{d} t}{t-z} .
$$

The value $f(t)$ refers to $(2.27)$ in which $H=\epsilon i$, and

$$
X_{0}(z)=\left(z^{2}-a^{2}\right)^{-1 / 2}
$$

which is a single-valued branch in the plane cut along $L(-a, a)$ and for which

$$
\lim _{|z| \rightarrow \infty} z X_{0}(z)=1
$$

Noting that

$$
X_{0}^{+}(t)=-X_{0}^{-}(t) \text { on } L .
$$

Equation (3.2) can be reduced to an integral along a clockwise closed contour $\Lambda$, which encircles $L$ (see Fig. 2)

$$
\begin{aligned}
I(z) & =\frac{1}{2}\left[\frac{1}{2 \pi i} \int_{-a}^{a} \frac{f(t)}{X_{0}^{+}(t)} \frac{\mathrm{d} t}{t-z}-\frac{1}{2 \pi i} \int_{-a}^{a} \frac{f(t)}{X_{0}^{-}(t)} \frac{\mathrm{d} t}{t-z}\right] \\
& =\frac{1}{2} \frac{1}{2 \pi i} \oint \frac{f(t)}{X_{0}(t)} \frac{\mathrm{d} t}{t-z} z_{0}, \bar{z}_{0} \text { are out of } \Lambda .
\end{aligned}
$$

Integrating, we obtain

$$
\begin{aligned}
I(z)= & \frac{1}{2 X_{0}(z)}\left[-\frac{M}{z-z_{0}}+\frac{\bar{N}}{\kappa\left(z-\bar{z}_{0}\right)}+\frac{\left(z_{0}-\bar{z}_{0}\right) \bar{M}+\bar{M}_{0}}{\kappa\left(z-\bar{z}_{0}\right)^{2}}-\Gamma+\frac{\bar{\Gamma}}{\kappa}+\frac{\bar{\Gamma}^{\prime}}{\kappa}+\frac{2 \mu \epsilon i}{\kappa}\right] \\
& +\frac{1}{2 X_{0}\left(z_{0}\right)} \frac{M}{z-z_{0}}-\frac{1}{2 X_{0}\left(\bar{z}_{0}\right)}\left[\frac{\bar{N}}{\kappa\left(z-\bar{z}_{0}\right)}+\frac{\left(z_{0}-\bar{z}_{0}\right) \bar{M}+\bar{M}_{0}}{\kappa\left(z-\bar{z}_{0}\right)^{2}}\right. \\
& \left.+\frac{\left(z_{0}-\bar{z}_{0}\right) \bar{M}-\bar{M}_{0}}{\kappa\left(z-\bar{z}_{0}\right)} \frac{\bar{z}_{0}}{\bar{z}_{0}^{2}-a^{2}}\right]+\frac{1}{2}\left(\Gamma-\frac{\bar{\Gamma}}{\kappa}-\frac{\bar{\Gamma}^{\prime}}{\kappa}-\frac{2 \mu \epsilon i}{\kappa}\right) z \\
& +\frac{1}{2}\left(M-\frac{\bar{N}}{\kappa}\right) .
\end{aligned}
$$


From (2.21), (2.22), (2.25), (3.1) and (3.7), it is seen that

$$
\begin{aligned}
\Phi(z)= & \frac{1}{2}\left[\frac{M}{z-z_{0}}+\frac{\bar{N}}{\kappa\left(z-\bar{z}_{0}\right)}+\frac{\left(z_{0}-\bar{z}_{0}\right) \bar{M}+\bar{M}_{0}}{\kappa\left(z-\bar{z}_{0}\right)^{2}}+\Gamma+\frac{\bar{\Gamma}}{\kappa}+\frac{\bar{\Gamma}^{\prime}}{\kappa}+\frac{2 \mu \epsilon i}{\kappa}\right] \\
& +\frac{1}{2} \frac{X_{0}(z)}{X_{0}\left(z_{0}\right)} \frac{M}{z-z_{0}}-\frac{1}{2} \frac{X_{0}(z)}{X_{0}\left(\bar{z}_{0}\right)}\left[\frac{\bar{N}}{\kappa\left(z-\bar{z}_{0}\right)}+\frac{\left(z_{0}-\bar{z}_{0}\right) \bar{M}+\bar{M}_{0}}{\kappa\left(z-\bar{z}_{0}\right)^{2}}\right. \\
& \left.+\frac{\left(z_{0}-\bar{z}_{0}\right) \bar{M}+\bar{M}_{0}}{\kappa\left(z-\bar{z}_{0}\right)} \frac{\bar{z}_{0}}{\bar{z}_{0}^{2}-a^{2}}\right]+\frac{1}{2}\left(\Gamma-\frac{\bar{\Gamma}}{\kappa}-\frac{\bar{\Gamma}^{\prime}}{\kappa}-\frac{2 \mu \epsilon i}{\kappa}\right) z X_{0}(z) \\
& +\frac{1}{2}\left(M-\frac{\bar{N}}{\kappa}\right) X_{0}(z) \\
\Omega(z)= & \frac{1}{2}\left[\frac{\kappa M}{z-z_{0}}+\frac{\bar{N}}{z-\bar{z}_{0}}+\frac{\left(z_{0}-\bar{z}_{0}\right) \bar{M}+\bar{M}_{0}}{\left(z-\bar{z}_{0}\right)^{2}}+\kappa \Gamma+\bar{F}+\bar{\Gamma}^{\prime}-2 \mu \epsilon i\right] \\
& -\frac{1}{2} \frac{X_{0}(z)}{X_{0}\left(z_{0}\right)} \frac{\kappa M}{z-z_{0}}+\frac{1}{2} \frac{X_{0}(z)}{X_{0}\left(\bar{z}_{0}\right)}\left[\frac{\bar{N}}{z-\bar{z}_{0}}+\frac{\left(z_{0}-\bar{z}_{0}\right) \bar{M}+\bar{M}_{0}}{\left(z-\bar{z}_{0}\right)^{2}}\right. \\
& \left.+\frac{\left(z_{0}-\bar{z}_{0}\right) \bar{M}+\bar{M}_{0}}{z-\bar{z}_{0}} \frac{\bar{z}_{0}}{\bar{z}_{0}^{2}-a^{2}}\right]+\frac{1}{2}\left(-\kappa \Gamma+\bar{\Gamma}+\bar{\Gamma}^{\prime}+2 \mu \epsilon i\right) z X_{0}(z) \\
& +\frac{1}{2}\left(-\kappa M+\bar{N}_{0} X_{0}(z) .\right.
\end{aligned}
$$

Substituting (3.8) and (3.9) into (2.34), then using the residue theorem, we obtain

$$
\begin{array}{r}
\epsilon=\frac{1}{\mu a^{2}} I_{m}\left\{\kappa M \left(z_{0}-\sqrt{\left.z_{0}^{2}-a^{2}\right)}-\bar{N}\left(\bar{z}_{0}-\sqrt{\left.\bar{z}_{0}^{2}-a^{2}\right)}-\left[\left(z_{0}-\bar{z}_{0}\right) \bar{M}\right.\right.\right.\right. \\
\left.\left.+\bar{M}_{0}\right]\left(1-\frac{\bar{z}_{0}}{\sqrt{z_{0}^{2}-a^{2}}}\right)\right\} \\
+\frac{1}{2 \mu} I_{m}\left(\kappa \Gamma-\bar{\Gamma}-\bar{\Gamma}^{\prime}\right) .
\end{array}
$$

For the special case of an arbitrary uniform stress state at infinity, we have

$$
X+i Y=0, \quad m=0 .
$$

Equations (3.8) and (3.9) become

$$
\begin{gathered}
\Phi(z)=\frac{1}{2}\left(\Gamma+\frac{\bar{\Gamma}}{\kappa}+\frac{\bar{\Gamma}^{\prime}}{\kappa}+\frac{2 \mu \epsilon i}{\kappa}\right)+\frac{1}{2}\left(\Gamma-\frac{\bar{\Gamma}}{\kappa}-\frac{\bar{\Gamma}^{\prime}}{\kappa}-\frac{2 \mu \epsilon i}{\kappa}\right) \frac{z}{\sqrt{z^{2}-a^{2}}} \\
\Omega(z)=\frac{1}{2}\left(\kappa \Gamma+\bar{\Gamma}+\bar{\Gamma}^{\prime}-2 \mu \epsilon i\right)+\frac{1}{2}\left(-\kappa \Gamma+\bar{\Gamma}+\bar{\Gamma}^{\prime}+2 \mu \epsilon i\right) \frac{z}{\sqrt{z^{2}-a^{2}}}
\end{gathered}
$$

which are in agreement with the solution in ref. [2].

Furthermore, for the special case of a uniaxial tension at infinity $\left(\sigma_{1}=\sigma, \sigma_{2}=0\right)$, we have

$$
\Gamma=\bar{\Gamma}=\frac{\sigma}{4} \quad \bar{\Gamma}^{\prime}=-\frac{1}{2} \sigma \mathrm{e}^{-2 i \alpha} .
$$

Equations (3.12) and (3.13) become

$$
\begin{aligned}
& \Phi(z)=\frac{1}{8}\left(1+\frac{1}{\kappa}\right) \sigma-\frac{\mathrm{e}^{-2 i \alpha}}{4 \kappa} \sigma+\frac{\mu \epsilon i}{\kappa}+\left[\frac{1}{8}\left(1-\frac{1}{\kappa}\right) \sigma+\frac{\mathrm{e}^{-2 i \alpha}}{4 \kappa}-\frac{\mu \epsilon i}{\kappa}\right] \frac{z}{\sqrt{z^{2}-a^{2}}} \\
& \Omega(z)=\frac{1}{8}(1+\kappa) \sigma-\frac{\mathrm{e}^{-2 i \alpha}}{4} \sigma-\mu \epsilon i+\left[\frac{1}{8}(1-\kappa) \sigma-\frac{\mathrm{e}^{-2 i z}}{4}+\mu \epsilon i\right] \frac{z}{\sqrt{z^{2}-a^{2}}}
\end{aligned}
$$


Equation (3.10) becomes

$$
\epsilon=\frac{\sigma \sin 2 \alpha}{4 \mu}
$$

which is in agreement with the result in ref. [2].

\section{Example 2}

A series of collinear periodical rigid lines are placed at $-a+2 n b \leqslant x \leqslant a+2 n b$ on the real axis. The stress state at $y= \pm \infty(z=x+i y)$ is given by $\sigma_{1}, \sigma_{2}$ and $\alpha$ (Fig. 3).

Making use of the conformal transformation of the $z$-plane onto the $\zeta$-plane which is given by eq. (2.37) (Fig. 4), eqs (2.19) and (2.20) are transformed into

$$
\begin{array}{cc}
{[\kappa \Phi(\xi)-\Omega(\xi)]^{+}+[\kappa \Phi(\xi)-\Omega(\xi)]^{-}=4 \mu \epsilon i} & |\xi| \leqslant \operatorname{tg} \frac{\pi a}{2 b}, \quad \eta=0 \\
{[\kappa \Phi(\xi)+\Omega(\xi)]^{+}-[\kappa \Phi(\xi)+\Omega(\xi)]^{-}=0} & |\xi| \leqslant \operatorname{tg} \frac{\pi a}{2 b}, \quad \eta=0 .
\end{array}
$$

The solution of eqs (3.18) and (3.19) can be written as

$$
\begin{gathered}
\Phi(\zeta)=\frac{C_{0}}{2 \kappa}+\frac{\mu \epsilon i}{\kappa}+\frac{C_{1}}{\kappa} \cdot \frac{\zeta}{\sqrt{\zeta^{2}-a_{1}^{2}}} a_{1}=\operatorname{tg} \frac{\pi a}{2 b} \\
\Omega(\zeta)=\frac{C_{0}}{2}-\mu \epsilon i-C_{1} \cdot \frac{\zeta}{\sqrt{\zeta^{2}-a_{1}^{2}}} .
\end{gathered}
$$

In the z-plane, we have

$$
\begin{gathered}
\Phi(\zeta)=\frac{C_{0}}{2 \kappa}+\frac{\mu \epsilon i}{\kappa}+\frac{C_{1}}{\kappa} \frac{\operatorname{tg} \frac{\pi z}{2 b}}{\sqrt{\operatorname{tg}^{2} \frac{\pi z}{2 b}-\operatorname{tg}^{2} \frac{\pi a}{2 b}}} \\
\Omega(z)=\frac{C_{0}}{2}-\mu \epsilon i-C_{1} \frac{\operatorname{tg} \frac{\pi z}{2 b}}{\sqrt{\operatorname{tg}^{2} \frac{\pi z}{2 b}-\operatorname{tg}^{2} \frac{\pi a}{2 b}}} .
\end{gathered}
$$

For a large value of $|y|$, we have

$$
\Phi(z)=\Gamma+0\left(\frac{1}{z^{2}}\right), \quad \Omega(z)=\bar{\Gamma}+\bar{\Gamma}^{\prime}+0\left(\frac{1}{z^{2}}\right) .
$$

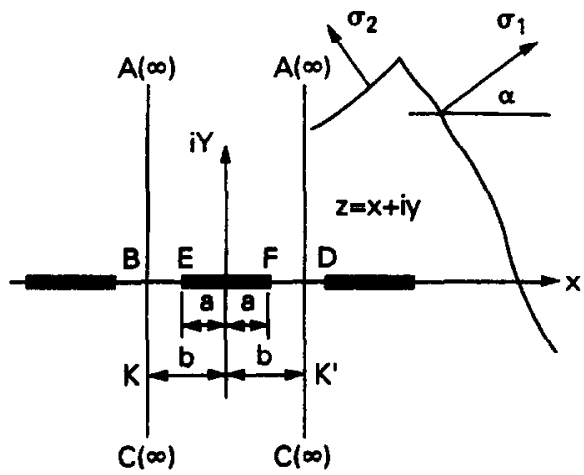

Fig. 3. Infinite plane with collinear periodical rigid lines.

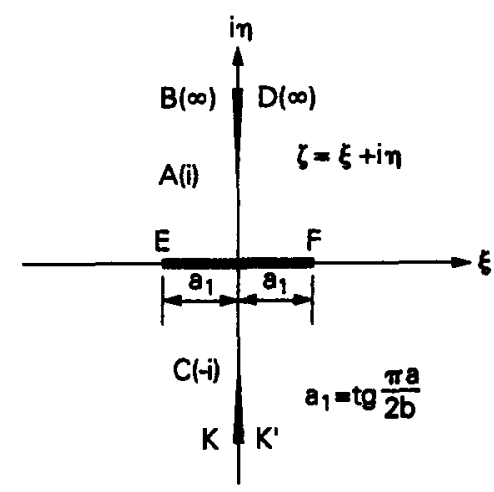

Fig. 4. Conformal map. 
From eqs (3.22)-(3.24), it is seen that

$$
\begin{gathered}
\frac{C_{0}}{2 \kappa}+\frac{\mu \epsilon i}{\kappa}+\frac{C_{1}}{\kappa} / \sec \frac{\pi a}{2 b}=\Gamma \\
\frac{C_{0}}{2}-\mu \epsilon i-C_{1} / \sec \frac{\pi a}{2 b}=\vec{\Gamma}+\bar{\Gamma}^{\prime}
\end{gathered}
$$

which give

$$
\begin{gathered}
C_{0}=\kappa \Gamma+\bar{\Gamma}+\bar{\Gamma}^{\prime} \\
C_{1}=\frac{1}{2}\left(\kappa \Gamma-\bar{\Gamma}-\bar{\Gamma}^{\prime}-2 \mu \epsilon i\right) \sec \frac{\pi a}{2 b} .
\end{gathered}
$$

Thus $\Phi(z)$ and $\Omega(z)$ take the forms

$$
\begin{aligned}
& \Phi(z)=\frac{1}{2 \kappa}\left(\kappa \Gamma+\bar{\Gamma}+\bar{\Gamma}^{\prime}+2 \mu \epsilon i\right)+\frac{1}{2 \kappa}\left(\kappa \Gamma-\bar{\Gamma}-\bar{\Gamma}^{\prime}-2 \mu \epsilon i\right) \frac{\sec \frac{\pi a}{2 b} \operatorname{tg} \frac{\pi z}{2 b}}{\sqrt{\operatorname{tg}^{2} \frac{\pi z}{2 b}-\operatorname{tg}^{2} \frac{\pi a}{2 b}}} \\
& \Omega(z)=\frac{1}{2}\left(\kappa \Gamma+\bar{\Gamma}+\bar{\Gamma}^{\prime}-2 \mu \epsilon i\right)-\frac{1}{2}\left(\kappa \Gamma-\bar{\Gamma}-\bar{\Gamma}^{\prime}-2 \mu \epsilon i\right) \frac{\sec \frac{\pi a}{2 b} \operatorname{tg} \frac{\pi z}{2 b}}{\sqrt{\operatorname{tg}^{2} \frac{\pi z}{2 b}-\operatorname{tg}^{2} \frac{\pi a}{2 b}}} .
\end{aligned}
$$

For the special case in which $\sigma_{1}=\sigma_{\infty}, \sigma_{2}=0, \alpha=0$, we have

$$
\Gamma=\bar{\Gamma}=\frac{\sigma_{\infty}}{4}, \quad \bar{\Gamma}^{\prime}=-\frac{\sigma_{\infty}}{2}, \epsilon=0 .
$$

Equations (3.29) and (3.30) become

$$
\begin{aligned}
& \Phi(z)=\frac{\sigma_{\infty}}{8}\left(1-\frac{1}{\kappa}\right)+\frac{\sigma_{\infty}}{8}\left(1+\frac{1}{\kappa}\right) \frac{\sec \frac{\pi a}{2 b}+\operatorname{tg} \frac{\pi z}{2 b}}{\sqrt{\operatorname{tg}^{2} \frac{\pi z}{2 b}-\operatorname{tg}^{2} \frac{\pi a}{2 b}}} \\
& \Omega(z)=\frac{\sigma_{\infty}}{8}(\kappa-1)-\frac{\sigma_{\infty}}{8}(\kappa+1) \frac{\sec \frac{\pi a}{2 b} \operatorname{tg} \frac{\pi z}{2 b}}{\sqrt{\operatorname{tg}^{2} \frac{\pi z}{2 b}-\operatorname{tg}^{2} \frac{\pi a}{2 b}}}
\end{aligned}
$$

which are in agreement with the results in ref. [3].

For the other special case in which $\sigma_{1}=0, \sigma_{2}=\sigma_{\infty}, \alpha=0$, we have

$$
\Gamma=\bar{\Gamma}=\frac{\sigma_{\infty}}{4}, \quad \bar{\Gamma}^{\prime}=\frac{1}{2} \sigma_{\infty}, \quad \epsilon=0 .
$$

Equations (3.29) and (3.30) become

$$
\begin{aligned}
& \Phi(z)=\frac{\sigma_{\infty}}{8}\left(1+\frac{3}{\kappa}\right)+\frac{\sigma_{\infty}}{8}\left(1-\frac{3}{\kappa}\right) \frac{\sec \frac{\pi a}{2 b} \operatorname{tg} \frac{\pi z}{2 b}}{\sqrt{\operatorname{tg}^{2} \frac{\pi z}{2 b}-\operatorname{tg}^{2} \frac{\pi a}{2 b}}} \\
& \Omega(z)=\frac{\sigma_{\infty}}{8}(\kappa+3)-\frac{\sigma_{\infty}}{8}(\kappa-3) \frac{\sec \frac{\pi a}{2 b} \operatorname{tg} \frac{\pi z}{2 b}}{\sqrt{\operatorname{tg}^{2} \frac{\pi z}{2 b}-\operatorname{tg}^{2} \frac{\pi a}{2 b}}} .
\end{aligned}
$$




\section{Example 3}

Consider a rigid line $L(-a, a)$ on which tractions are applied.

Since the remaining part of the plane is traction-free, the solution (2.28) becomes

$$
\Phi_{0}(z)=\frac{X_{0}(z)}{2 \pi i} \int_{-a}^{a} \frac{f(t)}{X_{0}^{t}(t)} \frac{\mathrm{d} t}{t-z}+C x_{0}(z)
$$

where

$$
f(t)=2 \mu \epsilon i / \kappa
$$

Hence

$$
\begin{gathered}
\Phi(z)=\Phi_{0}(z)=\frac{\mu \epsilon i}{\kappa}+\left(-\frac{\mu \epsilon i}{\kappa} z+C\right) \frac{1}{\sqrt{z^{2}-a^{2}}} \\
\Omega(z)=-\kappa \Phi(z) .
\end{gathered}
$$

Let $P+i Q$ be the resultant force of the tractions which are applied on the rigid line, and $m_{0}$ the sum of counterclockwise moments about the origin, of the tractions, the equilibrium conditions of the rigid line (2.33) and (2.34) are changed into

$$
\begin{gathered}
\oint_{\Lambda}[\Phi(z)-\Omega(z)] \mathrm{d} z=-P=i Q \\
\oint_{\Lambda} z\left[\Phi(z)-\Omega(z) \mathrm{d} z=-m_{0}\right.
\end{gathered}
$$

where $\Lambda$ is a clockwise closed contour encircling the rigid line.

The substitution of eqs (3.39) and (3.40) into eqs (3.41) and (3.42) yields

$$
\begin{aligned}
& C=-\frac{P+i Q}{2 \pi(1+\kappa)} \\
& \epsilon=\frac{\kappa m_{0}}{\pi(1+\kappa) \mu a^{2}} .
\end{aligned}
$$

\section{STRESS DISTRIBUTION AT THE RIGID LINE END}

It is of interest to examine the stress distribution in the immediate vicinity of the rigid line end. It is shown that the singular behavior of the stresses remains proportional to the inverse square root of $r$ (i.e. $r^{-1 / 2}$ ) as in the case of the immediate vicinity of a crack tip, where $r$ is the distance from the rigid line end. As an example, we discuss the case of a rigid line subjected a uniaxial tension at infinity $\left(\sigma_{1}=\sigma, \sigma_{2}=0\right)$. Introducing the polar coordinates $(r, \theta)$ at the rigid line end $+a($ Fig. 5 ), from eqs $(2.1),(2.10),(3.15)$ and (3.16), we obtain

$$
\begin{aligned}
& \sigma_{x}=\frac{\sigma}{8 \sqrt{2}} \sqrt{\frac{a}{r}} \cos \frac{\theta}{2}\left[\left(4+\kappa+\frac{3}{\kappa}\right)-2\left(1+\frac{1}{\kappa}\right) \sin \frac{\theta}{2} \sin \frac{3}{2} \theta\right] \\
& \sigma_{y}=\frac{\sigma}{8 \sqrt{2}} \sqrt{\frac{a}{r}} \cos \frac{\theta}{2}\left[\left(\frac{1}{\kappa}-\kappa\right)+2\left(1+\frac{1}{\kappa}\right) \sin \frac{\theta}{2} \sin \frac{3}{2} \theta\right] \\
& \tau_{x y}=\frac{\sigma}{8 \sqrt{2}} \sqrt{\frac{a}{r}} \sin \frac{\theta}{2}\left[\left(2+\kappa+\frac{1}{\kappa}\right)+2\left(1+\frac{1}{\kappa}\right) \cos \frac{\theta}{2} \cos \frac{3 \theta}{2}\right] \text { for } \alpha=0^{\circ}, \quad r \ll a
\end{aligned}
$$




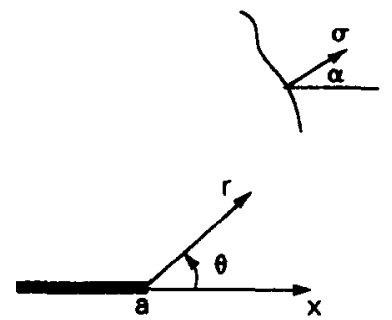

Fig. 5. Polar coordinates near a rigid line end.

$$
\begin{aligned}
& \sigma_{x}=\frac{\sigma}{8 \sqrt{2}} \sqrt{\frac{a}{r}} \cos \frac{\theta}{2}\left[\left(2+\kappa-\frac{3}{\kappa}\right)-2\left(1-\frac{1}{\kappa}\right) \sin \frac{\theta}{2} \sin \frac{3 \theta}{2}\right] \\
& \sigma_{y}=\frac{\sigma}{8 \sqrt{2}} \sqrt{\frac{a}{r}} \cos \frac{\theta}{2}\left[\left(2-\kappa-\frac{1}{\kappa}\right)+2\left(1-\frac{1}{\kappa}\right) \sin \frac{\theta}{2} \sin \frac{3 \theta}{2}\right] \\
& \tau_{x y}=\frac{\sigma}{8 \sqrt{2}} \sqrt{\frac{a}{r}} \sin \frac{\theta}{2}\left[\left(\kappa-\frac{1}{\kappa}\right)+2\left(1-\frac{1}{\kappa}\right) \cos \frac{\theta}{2} \cos \frac{3 \theta}{2}\right] \text { for } \alpha=45^{\circ}, \quad r \ll a .
\end{aligned}
$$

\section{REFERENCES}

[1] J. D. Eshelby, The determination of the elastic field of an ellipsoidal inclusion, and related problems. Proc. R. Soc. Lond. A241, 376-396 (1957).

[2] N. I. Muskhelishvili, Some Basic Problems of Mathematical Theory of Elasticity. Noordhoff, Leyden (1975).

[3] T. H. Hao and Y. C. Wu, Elastic plane problem of collinear periodical rigid lines. Engng Fracture Mech. 33, 979-981 (1989).

[4] C. P. Jiang, Z. Z. Zou, D. Wang and Y. W. Liu, A discussion about a class of stress intensity factors and its verification. Int. J. Fracture (to be published in 1991).

(Received 13 March 1990) 